\title{
Characterization of dual nano-size effects of ACC-cellulose nanofibrils on crystallization behavior of hydrophilic poly(vinyl alcohol)
}

\author{
Gento Ishikawa and Tetsuo Kondo* (1D
}

\begin{abstract}
This study attempts to clarify thermodynamic quantification on interaction between poly(vinyl alcohol) (PVA) and wood-derived cellulose nanofibrils (CNFs) obtained by aqueous counter collision (ACC) method. Aqueous mixtures of PVA/ACC-CNFs with various fiber widths were cast as the target materials. The interfacial interactions between the two components were characterized through thermodynamic evaluation of the crystalline PVA component as a probe in the cast mixture. As the result, surface properties of the ACC-CNFs found to reflect on the crystallization behavior of the interacted PVA component, resulting in dual nano-size effects of either diluent or nucleating agent. Melting point depression behaviors of the PVA component indicated that ACC-CNFs with thinner widths induced nucleation effects on PVA crystallization, whereas ACC-CNFs with ca. $100 \mathrm{~nm}$ in width encouraged diluent effects on PVA components. It is noted that this trend found to be reverse to the case for PVA/ACC-CNFs of bacterial nanocellulose previously reported.
\end{abstract}

Keywords: Janus amphiphilic nanocellulose, Melting point depression, Nano-size effect, Aqueous counter collision, Poly(vinyl alcohol)

\section{Introduction}

In the last two decades, nanocellulose, which is in general categorized into cellulose nanocrystals $(\mathrm{CNCs})$ and cellulose nanofibrils (CNFs), of width less than $50 \mathrm{~nm}$ and with an aspect ratio greater than 100, have attracted increasing attention in both basic and applied fields, e.g., in composites [1-6], paper and board [7], medicine [8], coatings [9-11], aerogels [12, 13], filtration [14], electronics [15], and rheological modifiers [16]. Nanocellulose has the following advantages: (1) it is produced from the most abundant sustainable natural source; (2) thermal stability in the range -200 to $200{ }^{\circ} \mathrm{C}$ [17], (3) a high crystalline elastic modulus and a low specific density [18], and (4) a desirable refractive index similar to those of common resins, enabling production of transparent

*Correspondence: tekondo@agr.kyushu-u.ac.jp

Graduate School of Bioresource and Bioenvironmental Sciences, Kyushu

University, West 5th, 744, Motooka, Nishi-ku, Fukuoka 819-0395, Japan nanocomposites. Acid hydrolysis and 2,2,6,6-tetramethylpiperidine-1-oxyl (TEMPO)-mediated oxidation are typical chemical processes that provide CNCs [19] and TEMPO-oxidized cellulose nanofibrils (TOCNs) [20, $21]$, respectively. In contrast, a grinding method [22, 23], which is a mechanical process, and the aqueous counter collision (ACC) method [24-26], which is a physicochemical process provide their characteristic CNFs.

Recently, it has been shown that the characteristics of the nanocellulose surface depend on the preparation process. Most nanocellulose generally possesses hydrophilic surfaces [27], but the ACC process can create more hydrophobic planes on nanocellulose surfaces $[25,28$, 29].

The ACC process, which was developed by Kondo et al. [24-26] involves repeated cycles of counter collisions using dual aqueous suspension jets containing samples under a high pressure (Fig. 1). The energy generated from collisions, typically at an ejection pressure of $200 \mathrm{MPa}$, 


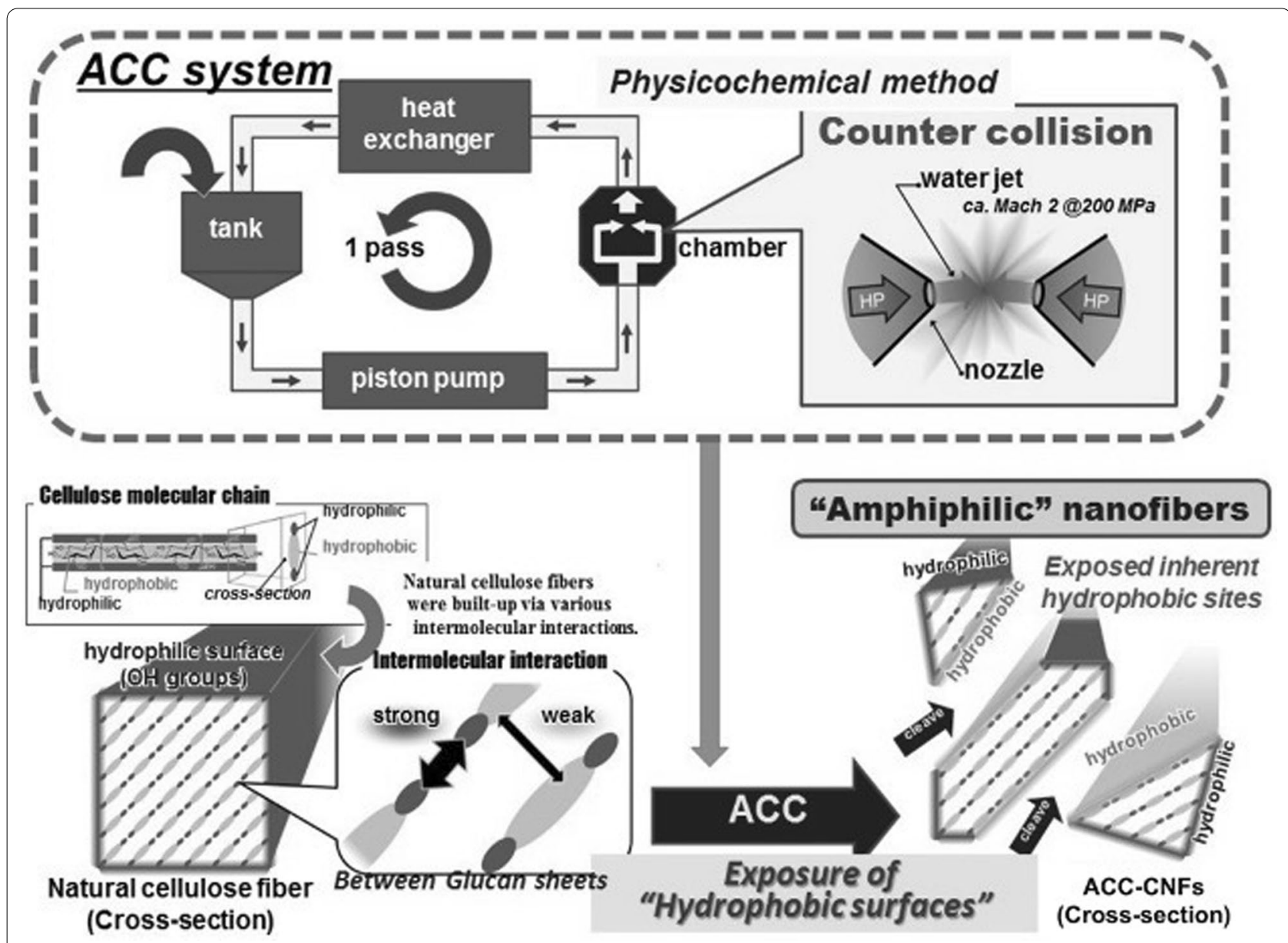

Fig. 1 ACC system for production of Janus-type amphiphilic CNFs

can be propagated as shock waves along elastic region in target samples, inducing destruction and/or continuous disruption of weak molecular interactions, such as van der Waals forces, and thus micro samples are pulverized to nanoscale samples. The destruction of interactions and the subsequent miniaturizing depend on the ejection pressure of the ACC water jets [26].

Native cellulose fibers have a hierarchical structure built on various interactions. A combination of hydrogen bonding and van der Waals forces stabilizes microsized cellulose fibers $(=$ pulp). In particular, hydrogen bonding among the equatorial hydroxy groups in individual anhydroglucose units provides glucan sheets with hydrophobic (=water-hating) faces. Then, macrofibers are fabricated by van der Waals interactions between the water-hating faces $[=(200)$ crystalline plane] of the glucan sheets. Destruction and disruption of the weaker van der Waals forces in microfibers produced by ACC expose the inherent water-hating faces on the resulting nanosized cellulose surfaces (see Fig. 1). In this way, the ACC process creates "amphiphilic CNFs with a Janus-type surface" having both hydrophobic and hydrophilic planes in a single nanofiber (ACC-CNF: see Fig. 1), and this enables their use to further extend towards surface modifiers, surfactants, emulsifiers, and dispersers [25, 30,31].

Recently, the Janus-type ACC-CNFs have been studied for usage of nanocomposites; for example, ACC-CNFs can be dispersed in a hydrophobic polypropylene polymer matrix [32, 33]. Here, clarification of the interfacial interactions between the filler and matrix is extremely necessary for the design of improved nanocomposites. In terms of the nano-size effect, it has generally been thought that smaller particles give better nanocomposite properties by increasing the surface area of the filler. The relationship between the mechanical properties and nanocomposite filler size has been extensively studied $[34,35]$. However, there have been few studies of the nano-size effect in nanocellulose composites, except in the case of ACC-CNFs [36, 37].

Advantages of ACC method are capable of providing CNFs of a desirable width from various cellulose sources, e.g. bacterial nanocellulose (BNC) $[25,36,37]$. Kose and 
Kondo reported nano-size effects of the ACC-BNCs in crystallization process of poly(lactic acid) (PLA) [36]. They pointed out that ACC-BNCs having widths within a certain nanoscale range were more effective nucleating agents than microfibers. Furthermore, in the nanoscale range in width, the ACC-BNCs with a smaller width found to exhibit a lower effect as nucleating agents for PLA; PLA crystallization in the presence of ACC-BNCs with $60 \mathrm{~nm}$ width was better than that in other systems with smaller ACC-BNCs. However, there was a certain problem that PLA does not always exhibit better interfacial miscibility to CNFs under directly mixing [38]. In our previous report [37], similar nano-size effect of ACC-BNCs on polymer crystallization was also observed at system of PVA/ACC-BNCs nanocomposite, and then interfacial interaction can be discussed quantitatively.

The aim of the current study was to quantitatively characterize the nano-size effects of ACC-CNFs from wood-biomass, not from bacterial source, by focusing on thermodynamic interactions [37], which gives some suggestions to use ACC-CNFs in applications as mentioned above. The interfacial interaction has been investigated by combination of wood ACC-CNFs and poly(vinyl alcohol) (PVA), which is crystalline polymer and expected to interact with cellulose molecules by hydrogen bonding [39, 40]. Namely, PVA crystallization behavior is employed as a probe for clarifying the effect with dependence on different fiber width at the nanoscale. Then, thermodynamic analyses have been carried out to investigate crystallization behavior of PVA components interfaced with the ACC-CNFs. The present results have shown that the ACC-CNF surface has a dual nano-size effect on PVA crystallization, but the reversal trend to ACC-bacterial CNFs reported previously [37]. It also corresponds to kinetic interpretation in crystallization process of the PVA component of the surface of ACC-CNFs.

\section{Materials and methods}

\section{Preparation of ACC-CNFs from microcrystalline cellulose}

Through this article, microcrystalline cellulose (MCC) (FUNACEL II ${ }^{\circledR}$, Funakoshi Co., Ltd., Tokyo, Japan) from wood pulp, which was suspended in water, was used directly without purification for ACC treatments. Namely, ACC-CNFs are indicated here as ACC-CNFs from wood pulp. The MCC was dispersed in ultrapure water at $0.4 \mathrm{wt} \%$. The number of collisions is expressed as a pass number (Pass). The ACC treatment conditions were $120 \mathrm{MPa}$ with 30 Pass, and $240 \mathrm{MPa}$ with 30 Pass. The suspensions obtained by ACC treatments were selectively fractionated to a fraction containing fibers with a defined width by centrifugation for $10 \mathrm{~min}$ at $25{ }^{\circ} \mathrm{C}$ under $400 \mathrm{~g}$ for the suspension obtained at $120 \mathrm{MPa}$, and under $51.1 \mathrm{~kg}$ for the suspension treated at $240 \mathrm{MPa}$, respectively. High-speed centrifugation of $51.1 \mathrm{~kg}$ for suspension treated at $240 \mathrm{MPa}$ was carried out under the purpose of preparation of more fine samples (see Additional file 1: Scheme S1).

\section{Transmission electron microscopy (TEM) for ACC-CNFs}

ACC-treated cellulose aqueous suspensions were dropped onto copper grids covered with formvar. The samples were negatively stained with $2 \%$ of uranyl acetate aqueous solution and air-dried before examination using TEM to determine the shapes and sizes of the fibers obtained by ACC treatments. For the observation, JEM1010 (JEOL Ltd., Tokyo, Japan) apparatus was used at $80 \mathrm{kV}$ of the accelerating voltage. After taking TEM images, according to previous research [25], the films were scanned and digitized to evaluate the average sizes of 50 cellulose fiber specimens in the images.

\section{Preparation of nanocomposites of ACC-CNFs with PVA}

Prior to mixing, PVA was saponified with $0.1 \mathrm{~mol} / \mathrm{L}$ sodium hydroxide aqueous solution to remove residual acetates. The saponification of PVA was $99.9 \%$. Aqueous suspensions of ACC-CNFs and PVA aqueous solution were prepared separately (see Additional file 1: Text S1).

A PVA solution (1.2 wt\%) was mixed with an aqueous suspension of ACC-CNFs by stirring overnight. The weight fraction of the treated cellulose in the mixture was controlled in the range $5-25 \%$. When added over $25 \%$, the endothermic peak was hard to be experimentally observed in differential scanning calorimetry. The mixture was poured into polyethylene cups, and degassed using a water aspirator in a warm bath at $50{ }^{\circ} \mathrm{C}$ for overnight. The suspension was dried in an oven at $50^{\circ} \mathrm{C}$ for casting.

\section{Measurement of nanocomposite film density}

The specific density $(d)$ of the cast film was measured to determine the volume fraction of cellulose fibers in the composite using pycnometry $[40,41]$. Density measurements were performed at $25{ }^{\circ} \mathrm{C}$ using $p$-xylene $(0.86 \mathrm{~g} /$ $\mathrm{cm}^{3}$, Wako Pure Chemical Industries, Ltd., Tokyo, Japan) and tetrachloromethane $\left(1.59 \mathrm{~g} / \mathrm{cm}^{3}\right)$.

\section{Volume fraction of ACC-CNF surface components [37]}

To investigate the effects of ACC-CNF surface interactions with PVA, the skin volume of the CNF components $\left(V_{\text {CNF-sur }}\right)$ was used as a thermodynamic index. The treated cellulose fibers were assumed to be " $n$-sided prisms" (Fig. 2) for investigating the effect of the crosssectional shape of the cellulose fibers produced by ACC treatment, whose schematic diagram is already shown in Fig. 1. 
Pre-derivation of the following Eq. (1): as a single ACCCNFs is assumed to have a polygonal cross-section, volume of $n$-sided prism is calculated as follows:

$$
V_{\mathrm{CNF}}=l R^{2} \frac{n}{2} \sin \frac{2 \pi}{n}
$$

Here, $l$ is length of nanofiber and $R$ is radius of $n$-polygon. When the thickness of a nanofibril skin is $t$, the surface skin volume is described by subtracting the inner volume from the entire volume of $n$-sided prism. Therefore,

$$
V_{\mathrm{CNF}-\mathrm{sur}}=l R^{2} \frac{n}{2} \sin \frac{2 \pi}{n}-(l-2 t)(R-t)^{2} \frac{n}{2} \sin \frac{2 \pi}{n} .
$$

Then, the surface skin volume fraction, $V_{\mathrm{CNF}-\text { sur }} / V_{\text {com }}$, was estimated using Eq. (3), i.e., (CNF number) $\times$ (surface skin volume of single $\mathrm{CNF}$ )/total volume of nanocomposite:

$$
\begin{aligned}
& V_{\mathrm{CNF}-\text { sur }} / V_{\mathrm{com}}=\mathrm{CNF} \text { number } \\
& \quad \times\left\{l R^{2} \frac{n}{2} \sin \frac{2 \pi}{n}-(l-2 t)(R-t)^{2} \frac{n}{2} \sin \frac{2 \pi}{n}\right\} / V_{\text {com }},
\end{aligned}
$$

where $V_{\text {com }}$ is the total volume of nanocomposite, $l$ is the nanocellulose length, $R$ is the cross-sectional radius, and $t$ is the effective surface thickness. The number of CNFs in a composite film, i.e., the CNF number, is assumed to be proportional to the volume fraction of CNF components. Based on this assumption, the CNF number can be given as

$$
\text { CNF number }=k \frac{V_{\text {cell }}}{V_{\text {com }}} .
$$

The parameter $k$ is the ratio of the weight of the composite body to that of a single CNF. When $W_{\text {com }}$ is the weight of the nanocomposite film, the volume fraction of the ACC-CNF surface can be obtained from Eqs. (3) and (4) as

$$
\begin{aligned}
& V_{\mathrm{CNF}-\mathrm{sur}} / V_{\text {com }}=k \frac{V_{\text {cell }}}{V_{\text {com }}} \\
& \quad \times\left\{l R^{2}-(l-2 t)(R-t)^{2}\right\} \frac{n}{2} \sin \frac{2 \pi}{n} / V_{\text {com }} .
\end{aligned}
$$

Then, dividing both sides by the parameter $k$, and subsequently transforming $V_{\text {cell }}$ and $V_{\text {com }}$ using their weights and densities, gives

$$
\begin{aligned}
\frac{V_{\mathrm{CNF}-\mathrm{sur}}}{k V_{\mathrm{com}}}= & \left\{l R^{2}-(l-2 t)(R-t)^{2}\right\} \\
& \times \frac{n}{2} \sin \frac{2 \pi}{n} \times \frac{\frac{f W_{\text {com }}}{d_{\text {cell }}}}{\left(\frac{W_{\text {com }}}{d}\right)^{2}} .
\end{aligned}
$$

Here, $f$ is the weight fraction of ACC-CNFs in the nanocomposite and $d_{\text {cell }}$ is the density of microcrystalline cellulose as $1.50 \mathrm{~g} / \mathrm{cm}^{3}$; Eq. (7) is finally obtained:

$$
\begin{aligned}
V_{\mathrm{NC}-\text { sur }} / k V_{\mathrm{com}}= & \left\{l R^{2}-(l-2 t)(R-t)^{2}\right\} \\
& \times \frac{n}{2} \sin \frac{2 \pi}{n} \times \frac{f / d_{\text {cell }}}{W_{\mathrm{com}} / d^{2}} .
\end{aligned}
$$

\section{Thermodynamic measurements to obtain equilibrium melting points}

Differential scanning calorimetry (DSC) was used to investigate the interfacial interactions between ACCCNFs and PVA. DSC for the nanocomposites was performed under a nitrogen atmosphere using a PerkinElmer DSC-7 instrument (PerkinElmer, Inc., Waltham, MA, USA). The instrument was calibrated with an indium standard. The equilibrium melting point of the PVA component mixed with ACC-CNFs was determined from the DSC measurements as follows: film specimens (5.00-6.67 $\mathrm{mg}$ ) were placed in aluminum sample pans and heated to $235{ }^{\circ} \mathrm{C}$; this temperature was maintained for $5 \mathrm{~min}$ to eliminate any PVA crystalline residues. The samples were quenched to the selected isothermal crystallization temperature and the temperature was held for $7 \mathrm{~h}$ to allow complete crystallization. During this step, the heat flow occurred by the crystallization of PVA

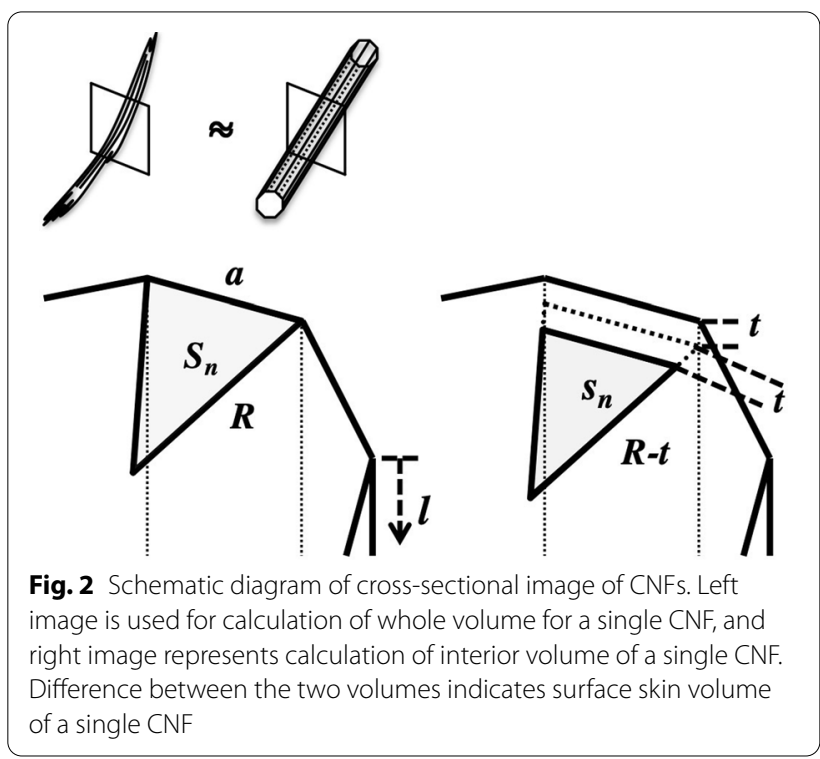


component in the nanocomposite was measured. The samples were then cooled to $20^{\circ} \mathrm{C}$. After isothermal crystallization of the sample, the melting point was measured using a heating rate of $10{ }^{\circ} \mathrm{C} / \mathrm{min}$. Hoffman-Weeks plots of the obtained melting points [42] were used to determine the equilibrium melting points of the films.

\section{Results and discussion}

\section{ACC-CNFs with different nanoscale widths}

The starting micro-sized wood-derived MCC samples were fractured into various nano-objects, depending on the ACC ejection pressure, which regulates collision energy to selectively cleave non-covalent bond in the microfibril under the purpose to control the width-size at the nanoscale. The pass number was needed to reduce amount of poorly-collided micro-targets for minimization of the nano-object distribution. TEM images of the ACC-treated MCC samples after centrifugation are shown in Fig. 3. The average ACC widths and lengths are listed in Table 1.

Figure 3 and the data in Table 1 show that three types of CNFs with different sizes were successfully prepared by ACC treatments and subsequent centrifugation [26]. Generally, for colloidal particles or proteins, the sedimentation time $(T)$ for centrifugation of spheres of diameter, diam, can be described by the Stokes equation [43]

$$
\begin{aligned}
& \frac{V}{T}=\frac{\operatorname{diam}^{2} \times\left(\rho_{\mathrm{p}}-\rho_{\mathrm{f}}\right) \cdot S \cdot g}{18 \eta}, \\
& V=\pi r^{2} L, \\
& S=\pi r^{2},
\end{aligned}
$$

where $V$ is the sedimentation volume, $\rho_{\mathrm{p}}$ is the particle density, $\rho_{\mathrm{f}}$ is the fluid density, $S$ is the sedimentation area, $g$ is the gravitational acceleration constant $\left(980.7 \mathrm{~cm} / \mathrm{s}^{2}\right)$, $\eta$ is the fluid viscosity, $L$ is the length of the centrifugation tube $(10.7 \mathrm{~cm})$, and $r$ is the tube diameter $(2.8 \mathrm{~cm})$. If the ACC-CNF can be regarded as a fine particle with the

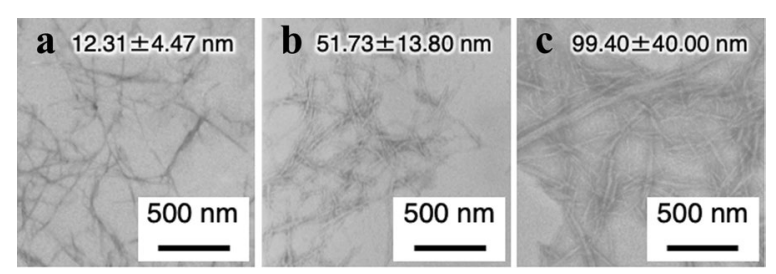

Fig. 3 TEM images of negatively stained ACC-treated MCC samples. a Width $12.31 \pm 4.47 \mathrm{~nm}$ (ACC-CNF-12), b width $51.73 \pm 13.80 \mathrm{~nm}$ (ACC-CNF-51), and c width $99.40 \pm 40.00 \mathrm{~nm}$ (ACC-CNF-99)
Table 1 Densities of ACC-CNFs/PVA composite films

\begin{tabular}{lrl}
\hline ACC-CNFs (averaged width $\times$ length) & $\begin{array}{l}\text { Weight fraction } \\
\text { of ACC-CNFs (\%) }\end{array}$ & $\begin{array}{l}\text { Composite } \\
\text { density }(\mathrm{g} / \\
\left.\mathrm{Cm}^{3}\right)\end{array}$ \\
\hline ACC-CNF-12 (12.3 nm $\times 230 \mathrm{~nm})$ & 5 & 1.492 \\
& 10 & 1.482 \\
& 15 & 1.471 \\
ACC-CNF-51 (51.7 nm $\times 500 \mathrm{~nm})$ & 20 & 1.462 \\
& 5 & 1.491 \\
& 10 & 1.481 \\
ACC-CNF-99 (99.4 nm $\times 1000 \mathrm{~nm})$ & 20 & 1.470 \\
& 10 & 1.459 \\
& 15 & 1.480 \\
& 20 & 1.468 \\
& 25 & 1.457 \\
\hline
\end{tabular}

same density as that of microcrystalline cellulose in the aqueous phase, under centrifugation at $F[\mathrm{G}]$, Eq. (8) can be transformed as follows:

$$
\operatorname{diam}^{2}=\frac{3.93 \times 10^{-3}}{T \times F} .
$$

The Stokes equation assumes sedimentation of ideal spherical particles, but asymmetric specimens do not necessarily follow this equation. However, these diam values are reasonable based on the average lengths in Table 1, which were obtained from TEM images. It should be noted here that Eq. (11), based on the Stokes equation, can be roughly applied in this case because of the anticipated sizes of the ACC-CNFs fractionated by centrifugation forces [26].

\section{Nano-size effects in kinetics of isothermal crystallization for PVA components mixed with ACC-CNFs PVA crystallinity after isothermal crystallization}

PVA crystallinity $\left(X_{c}\right)$ of the components after isothermal crystallization process on DSC was estimated using enthalpy of the fusion:

$$
X_{\mathrm{c}}=\frac{\Delta H_{\mathrm{obs}}}{\Delta H_{100}}
$$

where $\Delta H_{\text {obs }}$ is observed enthalpy of melting and $\Delta H_{100}$ is melting enthalpy of the perfect crystal which was estimated from enthalpy of the fusion for the repeating unit $1.64(\mathrm{kcal} / \mathrm{mol})[40]$.

Following by Eq. (12), crystallinity of neat PVA exhibited ca. 30-40\%. Both ACC-CNF-12 and ACC-CNF-51 contributed to enhancement on crystallinity of PVA up to ca. 55\%, which indicates that the two thinner ACC$\mathrm{CNFs}$ actually worked as a nucleating agent in the 


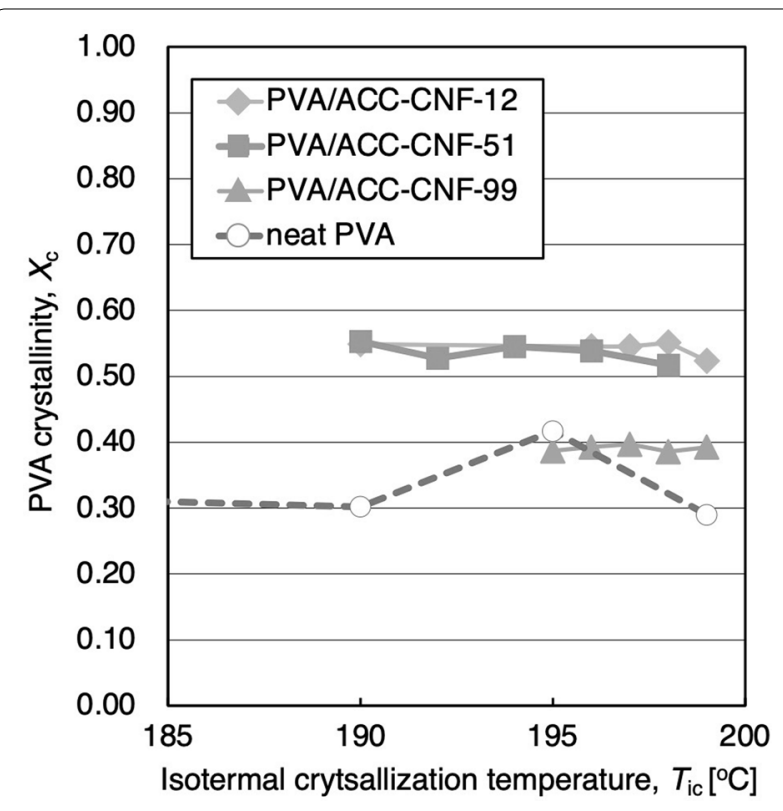

Fig. 4 PVA crystallinity versus isothermal crystallization temperature for PVA/ACC-CNFs with different width sizes on the nanoscale

nanocomposite system (Fig. 4). In contrast, PVA crystallinity for the sample with the thickest ACC-CNFs, ACCCNF-99, displayed below 40\%. This suggests that, the surface of ACC-CNF-99 has few potentials as nucleating agent.

\section{Crystallization behavior of PVA in nanocomposites}

Crystallization behavior of PVA components in nanocomposites of ACC-CNFs with different widths at the nanoscale was examined from kinetic viewpoints. Namely, the relative crystallinity of PVA $(X(t))$ was evaluated as a function of isothermal crystallization time $(t)$ :

$$
X(t)=\frac{A_{t}}{A_{\infty}} \times 100
$$

where $A_{t}$ is the exothermic areas under DSC thermograms from $t=A$ to $t=t$, while $A_{\infty}$ is the total areas surrounded by the thermograms and the baseline. Half time of crystallization $\left(t_{1 / 2}\right)$ was also defined as the time to reach $X(t)=50 \%$. Table 2 lists a set of representative $t_{1 / 2}$ data of the PVA/ACC-CNFs composites $(=95 / 5$ in weight), whose PVA component was isothermally crystallized at the different crystallization temperatures.

To compare values of the crystallization rate and the crystallization mechanism of the PVA component in the ACC-CNFs nanocomposites, Avrami theory was applied to the present isothermal crystallization processes. In the theory, relative crystallinity $(X(t))$ was described as follows:

$$
\begin{aligned}
& 1-X(t)=\exp \left(-K t^{n}\right), \\
& \ln (-\ln (1-X(t)))=n \ln t+\ln K,
\end{aligned}
$$

where $K$ is the overall kinetic rate constant, and $n$ is the Avrami exponent. These parameters are considered to be diagnostic to the mechanism of crystallization, because the two parameters, $K$ and $n$, are related to the crystallization rate and to the type of nucleation together with the geometry of the crystal growth, respectively. Here, Avrami parameters as listed in Table 2 were calculated

Table 2 Avrami parameters for isothermally crystallized PVA mixed with ACC-CNFs having different width at the nanoscale

\begin{tabular}{lllll}
\hline Sample & $T_{\text {ic }}\left({ }^{\circ} \mathrm{C}\right)$ & $n$ & $t_{1 / 2}(\min )$ & $-\ln K\left(\mathrm{~min}^{-n}\right)$ \\
\hline PVA/ACC-CNF-12 (95/5 in weight) & 190 & 2.123 & $181.93 \pm 0.01$ & 11.5 \\
& 196 & 2.137 & $174.29 \pm 0.01$ & 11.3 \\
& 197 & 2.143 & $175.59 \pm 0.01$ & 11.3 \\
& 198 & 2.122 & $164.02 \pm 0.01$ & 11.1 \\
PVA/ACC-CNF-51 (95/5 in weight) & 199 & 2.129 & $155.65 \pm 0.01$ & 11.0 \\
& 190 & 2.121 & $165.06 \pm 0.01$ & 11.1 \\
& 192 & 2.123 & $166.53 \pm 0.01$ & 11.1 \\
PVA/ACC-CNF-99 (95/5 in weight) & 194 & 2.142 & $175.53 \pm 0.01$ & 11.3 \\
& 196 & 2.139 & $173.96 \pm 0.02$ & 11.3 \\
& 198 & 2.160 & $181.03 \pm 0.01$ & 11.5 \\
neat PVA & 195 & 2.225 & $173.96 \pm 0.02$ & 12.0 \\
\end{tabular}


according to the Eqs. (14) and (15). The Avrami exponents of all samples are ranged in $2.1<n<2.3$, which reflected that crystal growth was confined in 2-D during the isothermal crystallization. The kinetic parameters in the system of PVA/ACC-CNFs nanocomposites indicated that, in each system of ACC-CNFs nanocomposites, PVA component crystallized faster than neat PVA. Namely, crystallization rate of PVA was accelerated by adding ACC-CNFs. In particular, ACC-CNF-12 and ACC-CNF-51 enhanced the PVA crystallization rate much faster than the thickest ACC-CNF-99 as shown in Table 2. As described above, ACC-CNF-12 and ACCCNF-51 contributes not only to enhancement on crystallinity of PVA, but to the PVA crystallization rate.

\section{Interactions between ACC-CNFs and PVA in nanocomposites \\ PVA melting point depression as a probe}

The nano-size effects of ACC-CNFs on the PVA components were also quantitatively investigated from the viewpoints of thermodynamic interactions. If a thermodynamic interaction occurs between the two components, the equilibrium melting point $\left(T_{\mathrm{m}}{ }^{\mathrm{eq}}\right)$ of the crystalline PVA component mixed with ACC-CNFs would be depressed.

In Fig. $5 \mathrm{a}$, the $T_{\mathrm{m}}{ }^{\text {eq }}$ values of the individual samples were obtained from Hoffman-Weeks plots [41] for isothermally crystallized PVA. Changes in the $T_{\mathrm{m}}{ }^{\text {eq }}$ values compared with that of neat PVA were clearly observed. The $T_{\mathrm{m}}{ }^{\text {eq }}$ for the individual composite samples were plotted against the cellulose volume fractions, as described in "Materials and methods" section.

The changing behaviors of the melting points observed using the samples of different widths, except in the case of ACC-CNF-99, did not show the changes typically observed for molecular blend systems [40-42]. Only increasing addition of ACC-CNF-99 resulted in increasing depression of $T_{\mathrm{m}}$ eq compared with that of neat PVA (Fig. 5b), indicating good miscibility between the two components and thus a diluent effect of the ACC-CNFs component. The opposite behavior was observed in the other two cases. The more ACC-CNF was added to the nanocomposites, the more the melting point was increased, resulting in increased melting temperatures. This indicates that these ACC-CNFs samples acted as nucleating agents, which agrees well to crystallization behavior as shown in Fig. 4. This is presumably because only the surfaces, and not the inner domains, of the individual ACC-CNFs components interacted with PVA. Therefore, Fig. 5a needs to be modified using "volume fraction parameter" [37] excluding interior volumes in the fibers.

Equation (3) was used to modify the volume fractions of the CNFs components [37]. The parameter $k$ was introduced to calculate the entire volume of the nanocomposite (Eq. 4). The parameter $k$ indicates the volume ratio of a single ACC-CNF compared with the entire nanocomposite body volume. The calculated $k$ values were nearly constant, indicating that $k$ does not necessarily depend on the ACC-CNF size. The volume fractions of the ACC-CNFs components were therefore modified by Eq. (7) using $k$. Additionally, the parameter $n$ represents
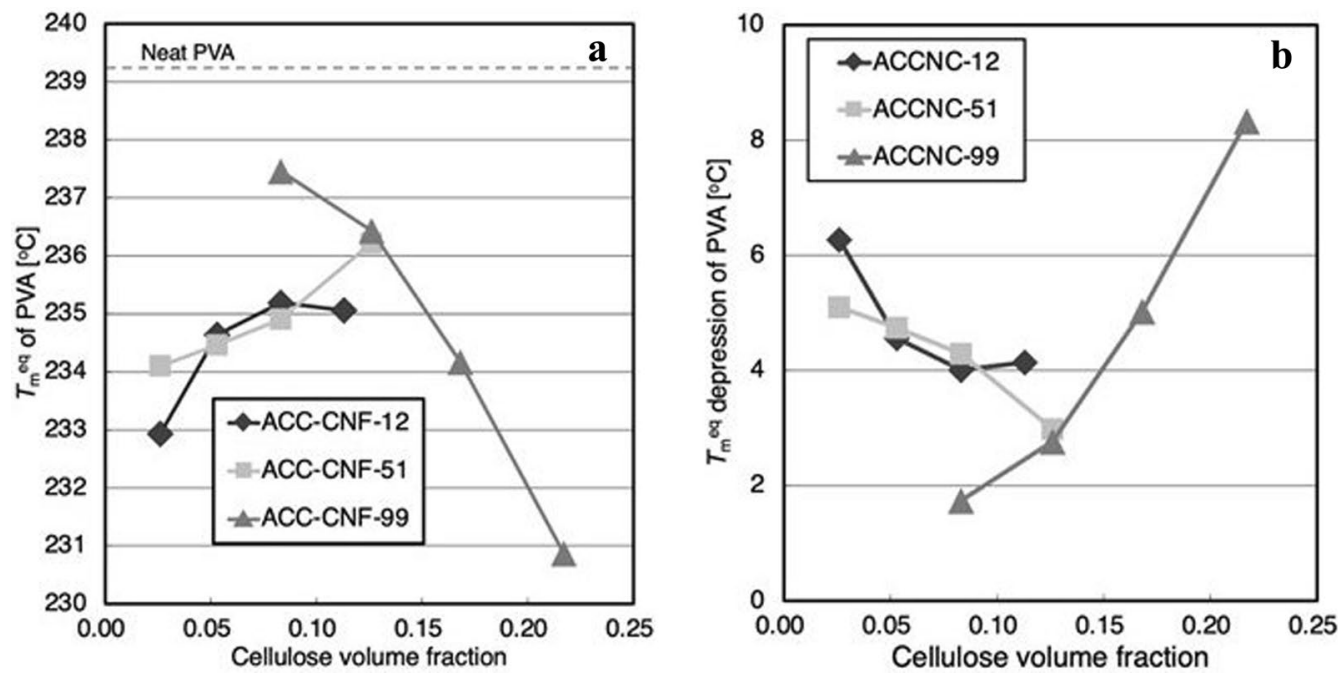

Fig. 5 a Equilibrium melting point $\left(T_{m}{ }^{\text {eq }}\right.$ ) of PVA components as function of cellulose volume fractions. $\mathbf{b}$ Relationship between the $T_{m}{ }^{\text {eq }}$ depression and cellulose volume fraction to be applied to Eq. (17) 
the cross section of a CNF as $n$-sided polygon. Recently, Tsuji et al. [29] reported that cross section of ACC-CNFs can be grasped in fact as trapezoid $(n=4)$, and thereafter $n=4$ can be used for Eqs. (3)-(7) to draw Fig. 6. Here, in Fig. 6, the horizontal axis shows the surface index on trapezoid $(n=4)$, which represents the surface skin volume fractions of the ACC-CNFs in the nanocomposites. Then, Fig. 6 exhibits that the changes in the melting point of PVA were clearly categorized into two behaviors corresponding to Fig. 5a, which depends on the ACCCNFs width. The $T_{\mathrm{m}}{ }^{\text {eq }}$ values of the PVA components mixed with ACC-CNF-12 and ACC-CNF-51 increased greatly with increasing in CNF surface volume fraction. In contrast, $T_{\mathrm{m}}$ eq decreased proportionally with increasing in total surface amounts of ACC-CNF-99 in the PVA matrix.

The cross sections of a single ACC-CNF were assumed to tetragon [ $n=4$ for Eq. (7)] [29].

This change depending on the fiber width can be explained as follows: the melting point changes of isothermally crystallized PVA samples suggest interfacial interactions among the components in the nanocomposites. Although amphiphilic Janus-type ACC-CNFs have hydrophobic planes, hydrophobic interaction never occurs without presence of water molecules. When considering about crystallization behavior for this PVA/ACC-CNFs system, depression of $T_{\mathrm{m}}{ }^{\text {eq }}$ for PVA reflected attractive interaction between PVA and hydrophilic plane of ACC-CNFs. For the two thinner

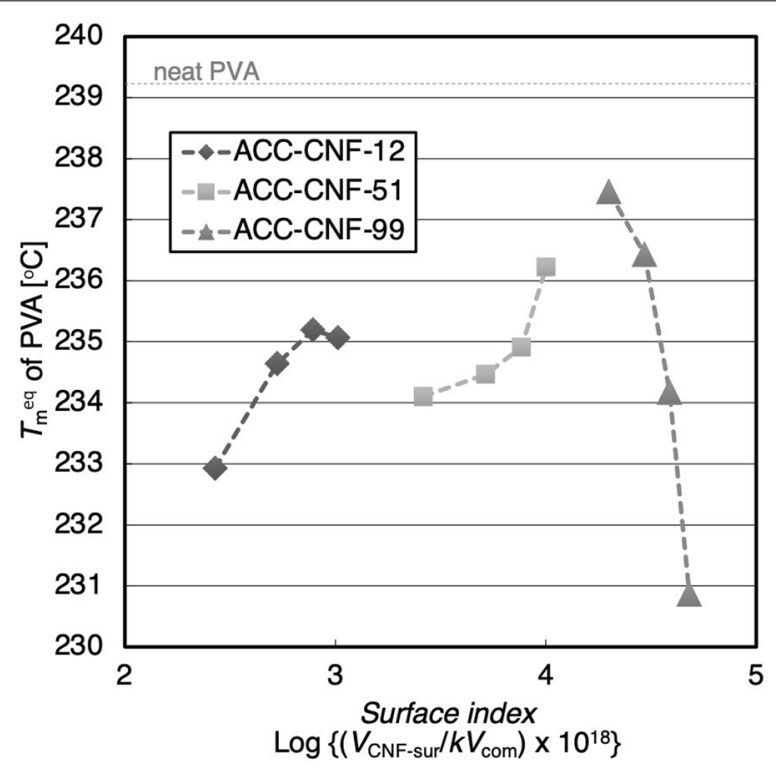

Fig. $6 T_{m}$ eq of PVA components against index representing skin volume fractions of ACC-CNFs with different widths. The cross-sections of a single ACC-CNF was assumed to tetragon $(n=4$ for Eq. (7)) [29] samples, i.e., ACC-CNF-12 and ACC-CNF-51, with PVA, the $T_{\mathrm{m}}{ }^{\text {eq }}$ increased with increasing surface skin volume fraction of ACC-CNFs (see Fig. 6). This behavior is considered to be the result of a nucleating effect. Namely, the surfaces of ACC-CNF-12 and ACCCNF-51 provide scaffolds for forming critical nucleus of hydrophilic PVA with lower free energy than the crystallization of neat system [44]. Hence, the thinner ACC-CNFs acted as "relatively" hydrophilic CNF. In contrast, increasing the surface volume fraction of the thicker ACC-CNF-99 enhanced the interactions between ACC-CNFs and PVA, resulting in $T_{\mathrm{m}}{ }^{\text {eq }}$ depression. The effect of the ACC-CNF-99 surface is assumed to be similar to a diluent effect.

These two conflicting results indicate that the surface characteristics of ACC-CNFs in contact with PVA differ depending on the width of the amphiphilic Janus-type ACC-CNFs $[25,28]$. In other words, the ACC-CNFs exhibit a unique dual nano-size effect on PVA, depending on its width and amphiphilic properties. Here, it should be noted that our previous study on ACC-treated bacterial nanocellulose (ACC-BNC) [37], which was derived from bacterial pellicle, composited with PVA exhibited the totally opposite phenomenon. Namely, thinner ACC-BNC displayed a diluent effect in the nanocomposites, whereas thicker one worked as a nucleating agent. This result indicated that, with increasing the width of ACC-BNC, free energy of forming critical nucleus of hydrophilic PVA on ACC-BNC became higher. In other words, ACC-BNC showed more "hydrophobic" potential than ACC-CNFs. This tendency was corresponded to other recent research of ACC-CNFs [29].

Another previous studies $[40,41]$ on cellulosic molecular blends showed that hydrophilic intermolecular interactions, such as hydrogen bonding, which occurred also between PVA and CNFs [37] and so as to nanocomposites of TEMPO-oxidized cellulose nanofibrils (TOCNs) [45]. Additionally, in the case of the ACC-CNFs, hydrophobic planes are exposed on the surface, which have few contributions on hydrogen bonding with PVA. The more detailed understanding on the coexistent effects of hydrophilicity and hydrophobicity on interactions with PVA components is required to clarify the mechanism of the unique nanosize effects of Janus-type amphiphilic ACC-CNFs.

In terms of the diluent effect, a cellulose molecule, i.e., the smallest cellulose component is miscible with PVA [40, 41]. In the present study, ACC-CNF-99 functioned in the same way as a cellulose molecule. The thinner ACC-MCC-12 and ACC-CNF-51 mainly acted as nucleating agents for PVA. Therefore, the width range indicates the nano-pulverization in ACC 
decreases hydrophilicity to some extent and increases the nucleating ability.

\section{Interaction parameters of ACC-CNFs as a diluent for PVA}

Unlike ACC-CNF-12 and ACC-CNF-51 exhibiting nucleating effects, ACC-CNF-99 acts as a diluent for PVA. To further understand this type of interaction between ACC-CNF-99 and PVA, the interaction parameter, $X_{12}$, was used, in an extension of the FloryHuggins approximation [46]. Scott proposed an equation for the thermodynamic depression of the melting point caused by a diluent [47]:

$$
1 / T_{\mathrm{m}}-1 / T_{\mathrm{m}}^{0}=-R \frac{V_{2 \mathrm{u}}}{\Delta H_{2 \mathrm{u}}}\left\{\frac{\ln v_{2}}{V_{2}}+\left(\frac{1}{V_{2}}-\frac{1}{V_{1}}\right)+\frac{\mathrm{B} v_{1}^{2}}{R T_{\mathrm{m}}}\right\},
$$

where $T_{\mathrm{m}}{ }^{0}$ is the melting point of neat PVA $(=512.36 \mathrm{~K})$; $T_{\mathrm{m}}$ is the observed $T_{\mathrm{m}}$ eq for PVA mixed with a diluent; $R$ is the gas constant $[1.987 \mathrm{cal} /(\mathrm{mol} \mathrm{K})]$; "1" and "2" refer to the non-crystallizable component and the crystalline component, respectively; $v_{1}$ and $v_{2}$ are the volume fractions; $V_{1}$ and $V_{2}$ are the molar volumes; $V_{2 \mathrm{u}}$ is the molar volume of a repeating unit of 2; and $\Delta H_{2 u}$ is the enthalpy of fusion per mole of repeating unit of 2 .

When ACC-CNFs and PVA are assumed to be the non-crystallizable component and crystalline component, Eq. (16) can be applied. Here, when $V_{1}$ and $V_{2}$ are $1 \times 10^{4} \mathrm{~cm}^{3} / \mathrm{mol}$ to $1 \times 10^{6} \mathrm{~cm}^{3} / \mathrm{mol}$, the entropy term in Eq. (16) can be entirely neglected [48]. The degree of polymerization of cellulose molecules in ACC-CNFs was estimated to be 200-250 because covalent bonds in the starting material (FUNACEL II ${ }^{\circledR}$ ) cannot be cleaved by the provided ACC energy; $V_{1}$ was estimated to be ca. $2 \times 10^{4} \mathrm{~cm}^{3} / \mathrm{mol}$. The $M_{w}$ of PVA was $31,000-50,000$ and $V_{2}$ was ca. $3 \times 10^{6} \mathrm{~cm}^{3} / \mathrm{mol}$. Eq. (16) can therefore be rearranged to Eq. (17), and the enthalpic contribution to the melting point depression can be evaluated:

$$
\Delta T_{\mathrm{m}}^{\mathrm{eq}}=-T_{\mathrm{m}}^{0} \frac{V_{2 \mathrm{u}}}{\Delta H_{2 \mathrm{u}}} B v_{1}^{2}
$$

$\Delta H_{2 \mathrm{u}} / V_{2 \mathrm{u}}$ for the PVA component was calculated to be $45.4 \mathrm{cal} / \mathrm{cm}^{3}$ [39]. Equation (17) is related to the parameter $B$, which indicates the interaction energy density characteristics of the two components. The interaction parameter, $\chi_{12}$, is described as

$$
\chi_{12}=\frac{B V_{1 \mathrm{u}}}{R T}
$$

In the case of the ACC-CNF-99/PVA nanocomposite, the volume fraction of the nanocellulose component, $v_{1}$, is considered to be the ratio of the cellulose volume to the entire composite volume. The nanocellulose
Table 3 Interaction energy density $(B)$ and interaction parameter $\left(X_{12}\right)$ for PVA/ACC-CNFs

\begin{tabular}{lll}
\hline & $\begin{array}{l}\text { Interaction energy density, } B \\
{\left[\mathrm{cal} / \mathrm{cm}^{3}\right]}\end{array}$ & $\begin{array}{l}\text { Interaction } \\
\text { parameter, } \\
X_{12}\end{array}$ \\
\hline ACC-CNF-99/PVA & -12.15 & -1.27 \\
$\begin{array}{c}\text { ACC-BNC (less than } \\
\text { 15 nm)/PVA [37] }\end{array}$ & -1.63 & -0.17 \\
$\begin{array}{c}\text { Cellulose/PVA molecular } \\
\text { blend (at 513 K, [40]) }\end{array}$ & -9.38 & -0.99 \\
\hline
\end{tabular}

Width size of CNF corresponds to diluent effect as the surface property. In Eq. (18), $V_{1 \mathrm{u}}=10^{7} \mathrm{~cm}^{3} / \mathrm{mol}$ and $512.36 \mathrm{~K}$ was employed

volume fraction can be used to calculate the values of $B$ and $X_{12}$, which are listed in Table 3.

Negative values of $B$ and $\chi_{12}$ indicate the presence of attractive interactions, such as hydrogen bonding and van der Waals forces, between CNFs and polymer molecules. Thermodynamic stability of interface between cellulosic and PVA components can be also estimated as magnitude of the above two parameter. The data in Table 3 indicate that the ACC-CNF-99 surface interacted stronger with the PVA component than cellulose molecules. Moreover, both of the thermodynamic indices of ACC-CNFs (from wood)/PVA system were much greater than those of ACC-BNC/PVA system. ACC-CNF-99 may prevent formation of stable nucleus of PVA than ACCBNC. The result could extend to the idea that, in terms of reinforcement for improving impact strength of polymer matrix, width-size effects as well as origin of ACCCNFs should become important factors to design CNFs composites.

\section{Conclusion}

Three types of CNFs with different widths were successfully prepared from microcrystalline cellulose by ACC treatments and subsequent centrifugation, without any chemical reaction.

The dual nano-size effects of ACC-CNFs were further investigated thermodynamically using PVA/CNF green composites. Namely, the ACC-CNF, which is assumed to have Janus-type of amphiphilicity, was mixed with PVA and the composite films were cast. The nano-size effects of ACC-CNFs on PVA in the nanocomposites were investigated thermodynamically based on the interfacial interactions between the two components. It was expected that the interactions would depress the equilibrium melting points, $T_{\mathrm{m}}$ eq, which were obtained from Hoffman-Weeks plots, of isothermally crystallized PVA. However, the changes in $T_{\mathrm{m}}$ eq with increasing surface volume fraction of the ACC-CNFs differed, depending on the width of the ACC-CNFs. The thinner ACC-CNFs 
acted as nucleating agents, but the thickest one, of width close to $100 \mathrm{~nm}$, acted as a PVA diluent. The results of dual nano-size effects indicated that there is an optimum size for achieving a nucleating effect at the nanoscale. In addition, the diluent effect of ACC-CNFs with amphiphilic planes is expected to promise a new design for a sort of miscible polymer matrices in their nanocomposites.

\section{Abbreviations}

ACC: Aqueous counter collision; BNC: Bacterial nanocellulose; CNCs: Cellulose nanocrystals; CNFs: Cellulose nanofibrils; DSC: Differential scanning calorimetry; MCC: Microcrystalline cellulose; PLA: Poly(lactic acid); PVA: Poly(vinyl alcohol); TEM: Transmission electron microscopy.

\section{Supplementary Information}

The online version contains supplementary material available at https://doi. org/10.1186/s10086-021-01957-9.

Additional file 1: Scheme S1. Preparation for ACC-CNFs having different widths at nanoscale.

\section{Acknowledgements}

None.

\section{Authors' contributions}

Gl: mainly performed entire experiments and preparation of the paper, TK: research plan through the study and preparation of the paper. Both authors read and approved the final manuscript.

\section{Funding}

Not applicable.

\section{Availability of data and materials}

Not applicable.

\section{Declarations}

\section{Competing interests}

The authors declare that they have no competing interests.

Received: 26 October 2020 Accepted: 10 March 2021

Published online: 20 March 2021

\section{References}

1. Wang B, Sain M (2007) Isolation of nanofibers from soybean source and their reinforcing capability on synthetic polymers. Compos Sci Technol 67:2521-2517

2. Fujisawa S, Saito T, Kimura S, Iwata T, Isogai A (2013) Surface engineering of ultrafine cellulose nanofibrils toward polymer nanocomposite materials. Biomacromolecules 14:1541-1546

3. Annamalai PK, Dangon KL, Monemian S, Foster EJ, Rowan SJ, Weder C (2014) Water-responsive mechanically adaptive nanocomposites based on styrene-butadiene rubber and cellulose nanocrystals-processing matters. ACS Appl Mater Interfaces 6:967-976

4. Iwamoto S, Yamamoto S, Lee SH, Endo T (2014) Solid-state shear pulverization as effective treatment for dispersing lignocellulose nanofibers in polypropylene composites. Cellulose 21:1537-1580

5. Sato A, Kabusaki D, Okumura H, Nakatani T, Nakatsubo F, Yano H (2016) Surface modification of cellulose nanofibers with alkenyl succinic anhydride for high-density polyethylene reinforcement. Compos A 83:72-79

6. Wang L, Okada K, Hikima Y, Ohshima M, Sekiguchi T, Yano H (2019) Effect of cellulose nanofiber (CNF) surface treatment on cellular structures and mechanical properties of polypropylene/CNF nanocomposite foams via core-back foam injection molding. Polymer 11:249

7. Lavoine N, Guillard V, Desloges I, Gontard N, Bras J (2016) Active bio-based food-packaging: diffusion and release of active substances through and from cellulose nanofiber coating toward food-packaging design. Carbohydr Polym 149:40-50

8. Jorfi M, Foster J (2015) Recent advances in nanocellulose for biomedical applications. J Appl Polym Sci 132:41719

9. Fukuzumi H, Saito T, Iwata T, Kumamoto Y, Isogai A (2009) Transparent and high gas barrier films of cellulose nanofibers prepared by TEMPOmediated oxidation. Biomacromolecules 10:162-165

10. Wu CN, Saito T, Fujisawa S, Fukuzumi H, Isogai A (2012) Ultrastrong and high gas-barrier nanocellulose/clay-layered composites. Biomacromolecules 13:1927-1932

11. Fukuzumi H, Fujisawa S, Saito T, Isogai A (2013) Selective permeation of hydrogen gas using cellulose nanofibril film. Biomacromolecules 14:1705-1709

12. Kobayashi Y, Saito T, Isogai A (2014) Aerogels with 3D ordered nanofiber skeletons of liquid-crystalline nanocellulose derivatives as tough and transparent insulators. Angew Chem, Int Ed 53:10394-10397

13. Nemoto J, Saito T, Isogai A (2015) Simple freeze-drying procedure for producing nanocellulose aerogel-containing, high-performance air filters. ACS Appl Mater Interfaces 7:19809-19815

14. Matsumoto M, Kitaoka T (2016) Ultraselective gas separation by nanoporous metal-organic frameworks embedded in gas-barrier nanocellulose films. Adv Mater 28:1765-1769

15. Fujisaki Y, Koga H, Nakajima Y, Nakata M, Tsuji H, Yamamoto T, Kurita T, Nogi M, Shimizu N (2014) Transparent nanopaper-based flexible organic thin-film transistor array. Adv Funct Mater 24:1657-1663

16. Isogai A, Kato Y (1998) Preparation of polyuronic acid from cellulose by TEMPO-mediated oxidation. Cellulose 5:153-164

17. Nishino T, Matsuda I, Hirao K (2004) All-cellulose composite. Macromolecules 37:7683-7687

18. Saito T, Kurame R, Wohlert J, Berglund LA, Isogai A (2013) An ultrastrong nanofibrillar biomaterial: the strength of single cellulose nanofibrils revealed via sonication-induced fragmentation. Biomacromolecules $14: 248-253$

19. Nickerson RF, Habrle JA (1947) Cellulose intercrystalline structure: study by hydrolytic methods. Ind Eng Chem 39:1507-1512

20. Saito T, Isogai A (2004) TEMPO-mediated oxidation of native cellulose. The effect of oxidation conditions on chemical and crystal structures of the water-insoluble fractions. Biomacromolecules 5:1983-1989

21. Saito T, Nishiyama Y, Putaux JL, Vignon M, Isogai A (2006) Homogeneous suspensions of individualized microfibrils from TEMPO-catalyzed oxidation of native cellulose. Biomacromolecules 7:1687-1691

22. Yano H, Nakahara S (2004) Bio-composites produced from plant microfiber bundles with a nanometer unit web-like network. J Mater Sci 39:1635-1638

23. Nakagaito AN, Yano H (2005) Novel high-strength biocomposites based on microfibrillated cellulose having nano-order-unit web-like network structure. Appl Phys A 80:155-159

24. Kondo T, Morita M, Hayakawa K, Onda Y (2005) US Patent 7,357,339

25. Kose R, Kasai W, Kondo T (2011) Switching surface properties of substrates by coating with a cellulose nanofiber having a high adsorbability. Sen-i Gakkaishi 67:153-168

26. Kondo T, Kose R, Naito H, Kasai W (2014) Aqueous counter collision using paired water jets as a novel means of preparing bio-nanofibers. Carbohydr Polym 112:284-290

27. Habibi Y (2014) Key advances in the chemical modification of nanocelluloses. Chem Soc Rev 43:1519-1542

28. Tsuboi K, Yokota S, Kondo T (2014) Difference between bamboo- and wood-derived cellulose nanofibers prepared by the aqueous counter collision method. Nord Pulp Pap Res J 29:69-76

29. Tsuji T, Tsuboi K, Yokota S, Tagawa S, Kondo T (2021) Characterization of amphiphilic Janus-type surface in cellulose nanofibril prepared by aqueous counter-collision. Biomacromolecules 21:620-628

30. Yokota S, Kamada K, Sugiyama A, Kondo T (2019) Pickering emulsion stabilization by using amphiphilic cellulose nanofibrils prepared by aqueous counter collision. Carbohydr Polym 226:115293

31. Tagawa S, Ishida K, Tsuji T, Kondo T (2021) Facile size evaluation of cellulose nanofibrils adsorbed on polypropylene substrates 
using fluorescence microscopy. Cellulose. https://doi.org/10.1007/ s10570-021-03759-0

32. Nodera A, Hashiba H, Tanaka H (2016) Japan patent application publication no. 2016-79311

33. Kondo T, Yokota S, Tanaka H (2017) Japan patent application publication no. 2017-234450

34. Muriithi B, Loy DA (2014) Proton conductivity of nafion/ex situ stöber silica nanocomposite membranes as a function of silica particle size and temperature. J Mater Sci 49:1556-1573

35. Li W, Yue J, Liu S (2012) Preparation of nanocrystalline cellulose via ultrasound and its reinforcement capability for poly(vinyl alcohol) composites. Ultrason Sonochem 19:479-485

36. Kose R, Kondo T (2013) Size effects of cellulose nanofibers for enhancing the crystallization of poly(lactic acid). J Appl Polym Sci 128:1200-1205

37. Ishikawa G, Kondo T (2017) Thermodynamic effect on interaction between crystalline phases in size-controlled ACC-bacterial nanocellulose and poly(vinyl alcohol). Cellulose 24:5495-5503

38. Pracella M, Haque MMU, Puglia D (2014) Morphology and properties tuning of PLA/cellulose nanocrystals bio-nanocomposites by means of reactive functionalization and blending with PVAc. Polymer 55:3720-3728

39. Sawatari C, Kondo T (1999) Interchain hydrogen bonds in blend films of poly(vinyl alcohol) and its derivatives with poly(ethylene oxide). Macromolecules 32:1949-1955

40. Nishio Y, Haratani T, Takahashi T (1989) Cellulose/poly(vinyl alcohol) blends: an estimation of thermodynamic polymer-polymer interaction by melting point depression analysis. Macromolecules 22:2547-2549
41. Kondo T, Sawatari C, Manley RS, Grey DG (1994) Characterization of hydrogen bonding in cellulose-synthetic polymer blend systems with regioselectively substituted methylcellulose. Macromolecules 27:210-215

42. Hoffman JD, Weeks JJ (1962) Melting process and equilibrium melting temperature of polychlorotrifluoroethylene. J Res Natl Bur Stand A 66(JAN-F):13-28

43. Van HKE, Johnson WC, Ho PS (1998) Principles of physical biochemistry. Prentice Hall Inc., Upper Saddle River

44. Urushihara T, Okada K, Watanabe K, Toda A, Kawamoto N, Hikosaka M (2009) Acceleration mechanism in critical nucleation of polymers by epitaxy of nucleating agent. Polym J 41:228-236

45. Endo R, Saito T, Isogai A (2013) TEMPO-oxidized cellulose nanofibril/ poly(vinyl alcohol) composite drawn fibers. Polymer 54:935-941

46. Flory PJ (1953) Principles of polymer chemistry. Cornell University Press, Ithaca, pp 568-571

47. Scott RL (1949) The thermodynamics of high polymer solutions. V. Phase equilibria in the ternary system: polymer 1-polymer 2-solvent. J Chem Phys 17:279-284

48. Imken RL, Paul DR, Barlow JW (1976) Transition behavior of poly(vinylidene fluoride)/poly(ethyl methacrylate) blends. Polym Eng Sci 16:593-601

\section{Publisher's Note}

Springer Nature remains neutral with regard to jurisdictional claims in published maps and institutional affiliations.

\section{Submit your manuscript to a SpringerOpen ${ }^{\circ}$ journal and benefit from:}

- Convenient online submission

- Rigorous peer review

- Open access: articles freely available online

- High visibility within the field

- Retaining the copyright to your article

Submit your next manuscript at springeropen.com 